\title{
Review: tricyclic antidepressants, capsaicin, gabapentin, and oxycodone are effective for postherpetic neuralgia
}

\author{
Alper BS, Lewis PR. Treatment of postherpetic neuralgia. A systematic review of the literature.J Fam Pract 2002 \\ Feb;51:121-8.

\section{QUESTION: In patients with postherpetic neuralgia (PHN), are any treatments effective in reducing pain or disability?}

\section{Data sources}

Studies were identified by searching Medline (1966 to October 2000) and the Cochrane Controlled Trials Registry with the terms postherpetic neuralgia, neuropathy, and pain; searching Current Contents, bibliographies of relevant studies, and the US Food and Drug Administration web site; and contacting authors and content experts.

\section{Study selection}

English language studies were selected if they were full reports of randomised controlled trials that included patients with PHN (history of herpes zoster, pain in the dermatomal distribution of the zoster rash, and pain persisting after resolution of the rash) and addressed relevant outcomes (pain resolution, pain severity, or quality of life).

\section{Data extraction}

2 reviewers independently reviewed trials for quality and extracted data on patient age and duration of PHN, type of treatment, treatment dosage and duration, results, and adverse effects.

\section{Main results}

27 trials were included. 6 trials evaluated topical treatments (follow up of $2 \mathrm{~d}$ to $6 \mathrm{wk}$ ). Of 2 trials that compared a lidocaine patch with placebo, 1 showed no difference and 1 showed a reduction in pain with lidocaine. 2 trials showed greater pain relief with capsaicin $0.075 \%$ cream than with placebo. 2 trials comparing benzydamine cream with placebo showed no difference between groups for pain relief.

12 trials evaluated oral treatments (follow up of $3 \mathrm{wks}$ to $6 \mathrm{mo}$ ). In 6 trials that assessed tricyclic antidepressants, amitriptyline was more effective than placebo (2 trials), lorazepam (1 trial), fluphenazine (1 trial), and maprotiline (1 trial) and was as effective as nortriptyline (1 trial); desipramine was more effective than benztropine (1 trial). Gabapentin was more effective than placebo for pain relief and improvement in sleep and quality of life (1 trial). Single placebo controlled trials of dextromethorphan, memantine, and acyclovir showed no difference between groups. Oxycodone was more effective than placebo for pain relief and disability scores (1 trial). 1 trial comparing tramadol with clomipramine with or without levomepromazine showed better pain relief with tramadol but no difference in pain intensity between groups.

9 trials evaluated other treatments (follow up of 8 wks to $2 \mathrm{y}$ ). Vincristine iontophoresis was no more effective than placebo in 1 trial and reduced pain in another small trial. Acupuncture was also not effective in 1 trial but had a short term effect in another small trial. Intrathecal methylprednisolone plus lidocaine was more effective than intrathecal lidocaine alone or no treatment ( 1 trial) and was more effective than epidural methylprednisolone (1 trial). 1 trial comparing a mixture of gangliosides with placebo showed improvement in pain and sleep scores with gangliosides but poor tolerability. Bupivacaine was more effective than was intravenous lidocaine for pain scores at up to 1 year (1 trial).

\section{Conclusions}

In patients with postherpetic neuralgia, tricyclic antidepressants have the strongest evidence of effectiveness in reducing pain or disability. Other treatments with some evidence of effectiveness include anticonvulsants (particularly gabapentin), capsaicin, and oxycodone.
Source of funding: Bureau of Health Professions Awards.

For correspondence: Dr B S Alper,

University of

Missouri-Columbia School of Medicine,

Columbia, Missouri,

USA

alperb@

health.missouri.edu.

\section{COMMENTARY}

The initial treatment choice for PHN usually lies between tricyclic antidepressants and anticonvulsants (the most widely studied being gabapentin). Although some have argued for making the choice based on the character of the pain (eg, antidepressants for burning pain and anticonvulsants for shooting pain), no strong evidence exists for this distinction. In the review by Alper and Lewis, the authors conclude that the strongest evidence for effectiveness in PHN exists for tricyclic antidepressants.

Approaching PHN as just one subset of neuropathic pain leads to a slightly different conclusion. In a previous systematic review that included trials of both $\mathrm{PHN}$ and diabetic neuropathy, Collins et al found that the numbers needed to treat to reduce neuropathic pain by $50 \%$ were identical for antidepressants and anticonvulsants ( 2.9 for both). ${ }^{1}$ Both types of drugs had a similar incidence of minor adverse effects (number needed to harm $[\mathrm{NNH}]$ of 2.7 for both). They defined a major adverse effect as one leading to withdrawal from treatment. Using this definition, they found an NNH of 17 for antidepressants. For anticonvulsants, no significant difference in major adverse events existed between active treatment and placebo.

Approaching pain from this perspective provides a counterpoint to the approach used by Alper and Lewis: it gives us an interesting example of the "lumping versus splitting" debate in research synthesis. By focusing on the type of pain rather than the cause, the review by Collins et al drew on a larger dataset. This led to more precise estimates of benefit and harm for the different classes of drugs. The clinical implications of the review by Alper and Lewis are that tricyclics are the first line treatment and anticonvulsants the second. The implication of the review by Collins $e t a l$, on the other hand, is that anticonvulsants may be the first choice on the basis of a better side effect profile. The limitation of that conclusion is that most trials report the number of side effects but not their severity. Many clinicians perceive the central nervous system side effects of anticonvulsants (giddiness, dizziness, and gait and visual disturbance) to be more severe than those of moderate dose tricyclics (dry mouth and hangover). In the absence of a head to head comparison, the choice of which to use first remains a matter of clinical judgment.

Henry McQuay, DM University of Oxford Oxford, $U K$

1 Collins SL, Moore RA, McQuay HJ, et al. Antidepressants and anticonvulsants for diabetic neuropathy and postherpetic neuralgia: a quantitative systematic review. J Pain Symptom Manage 2000;20:449-58. 